\title{
Feasibility and potential advantages using VMAT in SRS metastasis treatments
}

Maria Jose Perez-Calatayud ${ }^{1}$, Antonio Vicente Menendez ${ }^{3}$, Francisco Javier Celada-Alvarez ${ }^{2}$, Antonio Jose Conde-Moreno ${ }^{2}$, Mariola Bernisz ${ }^{2}$, Françoise Lliso², Vicente Carmona ${ }^{2}$, Jose Gimeno-Olmos ${ }^{2}$, Carlos Botella ${ }^{3}$, Jose Perez-Calatayud ${ }^{2,4}$

\author{
${ }^{1}$ Radiotherapy Department, Fundación Instituto Valenciano de Oncología, Valencia, Spain \\ ${ }^{2}$ Radiotherapy Department, Hospital La Fe, Valencia, Spain \\ ${ }^{3}$ Neurosurgery Department, Hospital La Fe, Valencia, Spain \\ ${ }^{4}$ Radiotherapy Department, Clinica Benidorm, Alicante, Spain
}

\section{ABSTRACT}

Background: Utilization of stereotactic radiosurgery (SRS) for brain metastases (BM) has become the technique of choice as opposed to whole brain radiation therapy (WBRT). The aim of this work is to evaluate the feasibility and potential benefits in terms of normal tissue (NT) and dose escalation of volumetric modulated arc therapy (VMAT) in SRS metastasis treatment. A VMAT optimization procedure has therefore been developed for internal dose scaling which minimizes planner dependence. Materials and methods: Five patient-plans incorporating treatment with frame-based SRS with dynamic conformal arc technique (DA) were re-planned for VMAT. The lesions selected were between 4-6 $\mathrm{cm}^{3}$. The same geometry used in the DA plans was maintained for the VMAT cases. A VMAT planning procedure was performed attempting to scale the dose in inner auxiliary volumes, and to explore the potential for dose scaling with this technique. Comparison of dose-volume histogram (DVH) parameters were obtained.

Results: VMAT allows a superior NT sparing plus conformity and dose scaling using the auxiliary volumes. The VMAT results were significantly superior in NT sparing, improving both the $V_{10}$ and $V_{12}$ values in all cases, with a $2-3 \mathrm{~cm}^{3}$ saving. In addition, VMAT improves the dose coverage $\mathrm{D}_{95}$ by about $0.5 \mathrm{~Gy}$. The objective of dose escalation was achieved with VMAT with an increment of the Dmean and the Dmedian of about $2 \mathrm{~Gy}$.

Conclusions: This work shows a benefit of VMAT in SRS treatment with significant NT sparing. A VMAT optimization procedure, based on auxiliary inner volumes, has been developed, enabling internal dose escalation.

Key words: SRS; VMAT; metastasis; clinical dosimetry; radiotherapy

Rep Pract Oncol Radiother 2021;26(1):119-127

\section{Introduction}

The frequency of brain metastasis (BM) in oncologic patients is significant; an incidence higher than $20 \%$ has been reported and is the most common intracranial disease [1-4]. Radiotherapy and surgery are the two main treatment options for these lesions. Radiation therapy (RT) has been demonstrated to be especially beneficial for these patients [5-11].

It has been reported in the literature that treatment outcomes and toxicity are improved with the use of stereotactic radiosurgery (SRS) or hypofractionated stereotactic radiotherapy (HFSRT) instead

Address for correspondence: Maria Jose Perez-Calatayud, 1Radiotherapy Department, Fundación Instituto Valenciano de Oncología, Valencia, Spain; e-mail: mariajose.perezcalatayud@gmail.com

This article is available in open access under Creative Common Attribution-Non-Commercial-No Derivatives 4.0 International (CC BY-NC-ND 4.0) license, allowing to download articles and share them with others as long as they credit the authors and the publisher, but without permission to change them in any way or use them commercially 
of whole brain radiation therapy (WBRT) [12]. Utilization of SRS for BM has increased, prompting a reassessment of WBRT $[13,14]$, and allowing for the possibility of repeated treatments of sequentially diagnosed BM [15]. Barbour et al. [16] have analyzed the practice patterns of SRS and WBRT for BM in the USA. They concluded that the use of WBRT had decreased while SRS utilization had doubled and become the first RT option for this group of patients.

Intracranial SRS was performed generally with a Gamma Knife, however, during the last decade, robotic radiosurgery (CyberKnife) and modern linacs have also been used [17]. Usually, the SRS treatment of metastasis using linac is based on dynamic conformal arcs (DA), using a non-coplanar approach [18]. In recent years, volumetric modulated arc therapy (VMAT) has been implemented in linacs [19] and applied also in HFSRT and SRS [20].

Currently, our departments are applying VMAT in SRS for benign tumors of the skull base and in most arteriovenous malformations (AVM), taking advantage of a higher conformation and dose increase of a well-defined volume (non-published data). Typically, SRS DA is applied in cases of small size metastasis. Usually, non-coplanar DA are used, the Prescribed Dose (PD) ranging from 16 to $22 \mathrm{~Gy}$, depending on the lesion size. For larger or irregularly shaped metastases, the use of VMAT allows a better conformational technique.

In most centers, SRS in BM is commonly performed both frame or frameless. The criteria are the relevance of the normal tissue (NT) involved because of the margin from the gross tumour volume (GTV) to the planning target volume (PTV) (set-up) [21]. Examples of cases chosen for frame-based SRS would be lesions located in an eloquent area, near the brainstem or the optic pathway. Frameless procedures are more efficient for departmental reasons: mask preparation, CT acquisition, MRI co-registration, contouring, treatment planning and VMAT experimental verification. In contrast with a frameless case, SRS with a frame implies that the whole procedure should be performed during a working day.

The normal brain tissue is evaluated by the $\mathrm{V}_{12}$, volume of NT (brain minus GTV) irradiated with a dose greater than or equal to $12 \mathrm{~Gy}$, as recommended elsewhere [22-26]. If the tumor(s) location is not so critical, NT $V_{12}$ up to $10 \mathrm{~cm}^{3}$ is acceptable.
However, for BM within (or very close to) eloquent areas, lower limits are preferred: optimal $4 \mathrm{~cm}^{3}$, acceptable $4-6 \mathrm{~cm}^{3}$, mandatory $<6 \mathrm{~cm}^{3}$. Nevertheless, in the decision-making process, these general guidelines are not a substitute for a thorough, case by case analysis. According to these criteria, the decision is typically made between HFSRT or SRS and the use of a frame or mask.

The aim of this work is to evaluate the feasibility and potential benefits in terms of NT and dose escalation of VMAT in SRS metastasis treatment. To accomplish this, a VMAT optimization procedure has been developed for internal dose scaling which minimizes planner dependence.

\section{Materials and methods}

In order to evaluate the potential benefit of VMAT, plans for five patients with lesions between 4 and $6 \mathrm{~cm}^{3}\left(4.3,4.5,5.6,5.7\right.$ and $\left.6.7 \mathrm{~cm}^{3}\right)$ were retrospectively evaluated. These patients were previously treated with a frame-based SRS DA technique using 4 to 6 non-coplanar arcs.

\section{DA plan characteristics}

First, the Brainlab (BrainLAB AG, Feldkirchen, Germany) headframe is placed under local anesthesia, with mild sedative medication and analgesia. Next, on a Philips CT scanner, Brilliance Big Bore (Philips HealthCare, Best, Netherlands) a cranial CT is obtained with the finer resolution available compatible with the required quality for contouring, that is $0.8 \mathrm{~mm}$. Contrast media (50 $\mathrm{cm}^{3}$ with a lapse for image acquisition of $45 \mathrm{~s}$ ) is administered to enhance the tumor visibility. Image co-registration of CT and MRI (done within 2 weeks previous to SRS) is performed in the Treatment Planning System (TPS) iPlan (v4.5.5. from Brainlab). MRI (Signa HDtx from General Electric, Milwaukee, WI, USA) characteristics are: MPRGE T1 with IVC media, overlapping slices of $0.5 \mathrm{~mm}$ width; for tumors related with the CSF pathways, ventricles or skull base a selective $\mathrm{T} 2$ technique is employed (FIESTA or CISS). Contouring of GTV and organs at risk (OAR) are also performed in iPlan; GTV is assumed as PTV and then no extra margin is given $[12,28,29]$.

From 4 to 6 non-coplanar 6 MV DA are defined in iPlan, depending on the lesion laterality. Typical couch angle separation is 30 to 40 degrees with 
no opposing arcs. The gantry angle range of arcs is about 120 to 140 degrees. In all cases, the collimator angle is adjusted by TPS to minimize the gap between MLC and the GTV contour along the beam eye views. A margin of $1 \mathrm{~mm}$ is applied to MLC. Furthermore, a grid resolution of $1 \mathrm{~mm}$ for the calculation procedures is set. The pencil beam algorithm is used. The beams are administered in a linac TrueBeam v. 2.5 (Varian, Palo Alto, CA) with high definition MLC (inner width of $2.5 \mathrm{~mm}$ at the isocenter). Once the frame is fixed to the couch, final patient set-up is performed using a $6 \mathrm{D}$ couch after CBCT vs. CT matching, although the target positioners from the stereotactic guide are used only as a starting alignment position.

The planning aim is to have a $\mathrm{D}_{95}$ (being $\mathrm{D} x$ the minimum dose to the $\mathrm{x} \%$ of the target volume in Gy) coverage of the GTV, which will receive the PD, keeping the PTV homogeneity within 110-115\%. The PD is established according to the lesion volume, due to the impact on the NT dose. The dose vs volume typically applied is: $21 \mathrm{~Gy}, 19.5 \mathrm{~Gy}, 18 \mathrm{~Gy}$ and $16 \mathrm{~Gy}$ for around $1 \mathrm{~cm}^{3}, 2 \mathrm{~cm}^{3}, 4 \mathrm{~cm}^{3}$ and $6 \mathrm{~cm}^{3}$, respectively.

\section{VMAT arcs plan characteristics}

The VMAT plans were optimized and calculated in Eclipse v. 15.5 (Varian, Palo Alto, CA) using an RX 6MV Flattening Filter Free (FFF). An AAA algorithm (v. 15.511) was used with a $1 \mathrm{~mm}$ grid resolution and a VMAT optimization done with the PO algorithm (v. 15.5.11). The arcs (couch and gantry range) used in the DA plans were maintained in the VMAT planning case. All structures (included the inner auxiliary volumes) were defined as "high resolution" volumes in Eclipse. A $20 \mathrm{~mm}$ ring was built around the target to evaluate the normal brain tissue dose.

VMAT planning was performed in each case in an attempt to scale the dose in the inner volumes arranged concentrically. The aim was to evaluate if the inner dose escalation by using VMAT is compatible with NT sparing. Inner auxiliary volumes were successively defined by applying a negative 1 $\mathrm{mm}$ margin. For example, in a $4.5 \mathrm{~cm}^{3}$ case, the inner resulting volumes were $\mathrm{C} 1=2.7 \mathrm{~cm}^{3}, \mathrm{C} 2=1.5$ $\mathrm{cm}^{3}$ and $\mathrm{C} 3=0.7 \mathrm{~cm}^{3}$. To explore the potential for dose scaling, an increment of 2 Gy was intended for each inner volume. For example, in the former 4.5 $\mathrm{cm}^{3}$ case in which the PD was $18 \mathrm{~Gy}$, the intended dose for C1, C2 and C3 was 20 Gy, 22 Gy and 24 Gy respectively, keeping the maximum as an extra $2 \mathrm{~Gy}$ increment, that is $26 \mathrm{~Gy}$, which should be within the inner volume $\mathrm{C} 3$. For one of the cases, due to the small volume, just two inner volumes were defined. The selection of the $2 \mathrm{~Gy}$ increment value along the inner auxiliary volumes was arbitrary, with the aim solely to explore the scaling capabilities. No other values were tested.

To minimize planner-dependence in the optimization procedure, a general approach has been developed and applied to all 5 cases presented in this work. To help in the optimization process, auxiliary differential shells were obtained by boolean subtractions, these are GTV-C1, C1-C2 and $\mathrm{C} 2-\mathrm{C} 3$; the aim was to guide the overdose volumes through the GTV center. Due to the small resulting volumes, in some cases with Eclipse the structure obtained was not connected. Nevertheless, optimization worked correctly because the objective was to avoid an excessive maximum.

The specific parameters used in the optimization are summarized in Table 1 . The priority numbers are relative, the higher values assigned to the NT constraints together with the PD are taken from the DA plan case. The coverage of GTV and the inner volumes had an intermediate priority, with those assigned to the upper dose value in each volume being lower. The differential shells had only the condition of the maximum dose, with lower priority, attempting to avoid a potential deviation of the maximums from the innermost volume. A maximum of 1 Gy additional to the coverage of the subsequent inner volume is then selected. Examples of the defined inner volumes are shown in Figure 1. RefDA10 and RefDA12, the NT $V_{10}$ and $V_{12}$ resulted in the DA plan respectively, are the baseline values for NT in the optimization; during the procedure and looking at their competition in the objective function against the GTV coverage, these values were decreased until the $\mathrm{D}_{95}$ of GTV received the PD.

\section{Plan comparisons}

The DA plans performed in iPlan TPS were exported and recalculated in Eclipse where both dose-volume histograms (DVHs) were evaluated and compared. It was done trying to avoid the critical issue in the volume calculation for very small structures, because we noticed small differences 
Table 1. Optimization parameters in Eclipse

\begin{tabular}{|l|c|c|c|c|}
\hline Structure & Constraint type & Vol (\%) & PD +8 & 150 \\
\hline GTV & Superior & 0 & PD & 200 \\
\hline C1 & Inferior & 98 & PD +8 & 150 \\
\hline & Superior & 0 & PD +2 & 200 \\
\hline C2 & Inferior & 98 & PD +8 & 150 \\
\hline & Superior & 0 & PD +4 & 200 \\
\hline C3 & Inferior & 98 & PD +8 & 150 \\
\hline & Superior & 0 & PD +6 & 200 \\
\hline GTV-C1 & Inferior & 98 & PD +3 & 100 \\
\hline C1-C2 & Superior & 0 & PD +5 & 100 \\
\hline C2-C3 & Superior & 0 & PD + 7 & 100 \\
\hline Normal tissue & Superior & 0 & 10 & 12 \\
\hline
\end{tabular}

$P D$ - the prescribed dose, taken from the dynamic conformal arcs (DA) plan of this case, similarly the $V_{10}$ (RefDA10) and $V_{12}$ (RefDA12) values for normal tissue; GTV - gross tumour volume
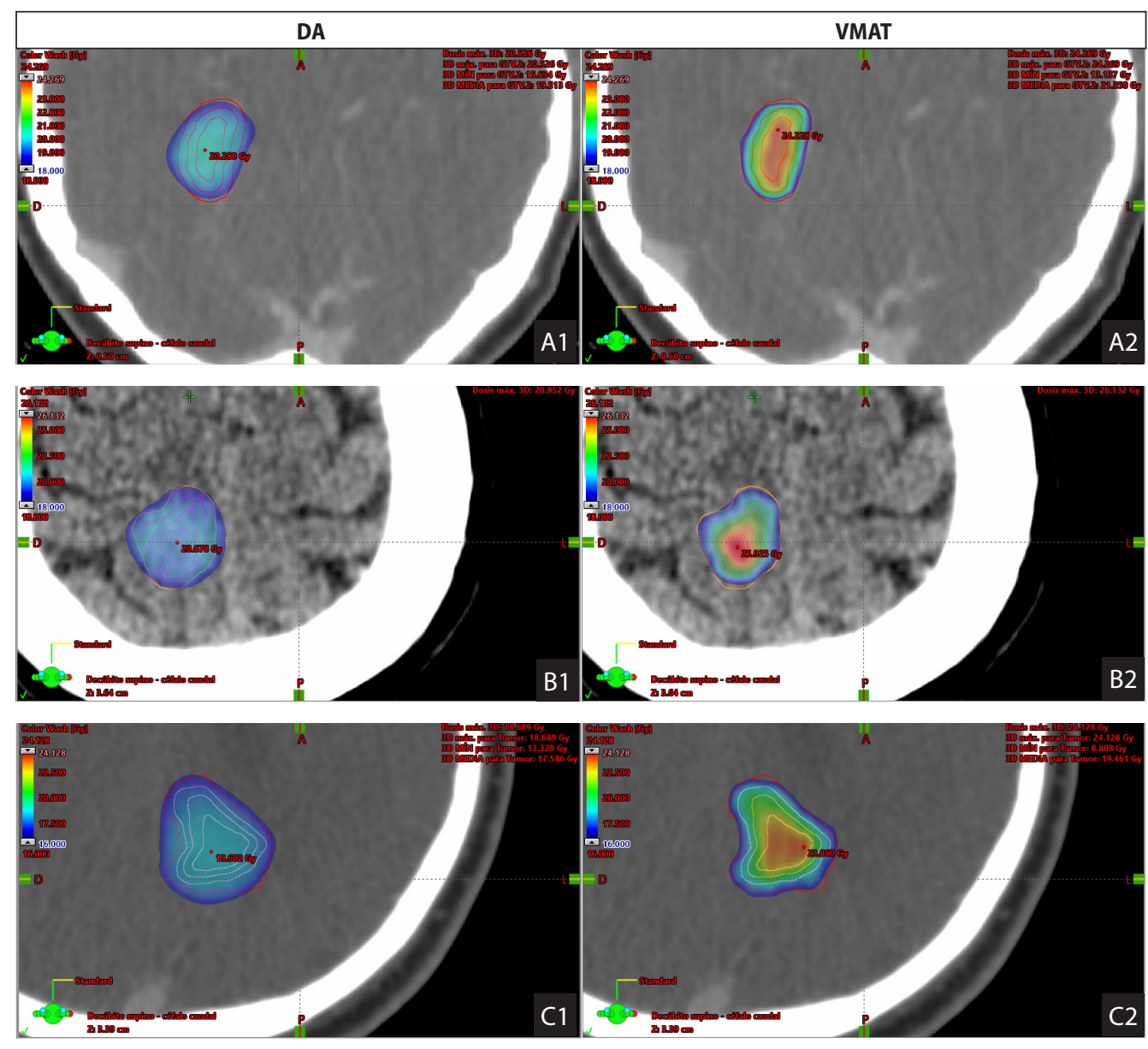

Figure 1. Dose distribution comparison DA vs. VMAT for three test cases: (A), case \#1 small $\left(4.31 \mathrm{~cm}^{3}\right)$, (B), case \#3 medium $\left(5.55 \mathrm{~cm}^{3}\right)$ and $(\mathbf{C})$ case \#6 large $\left(6.66 \mathrm{~cm}^{3}\right.$ lesion size). Inner auxiliary volumes are shown for each case. Lower isodose level corresponds to the prescription dose 
between both TPSs. Target coverage in DA was $\mathrm{D}_{95}$ equal to the PD, in the VMAT plans the optimization was done to fulfill at least this coverage, keeping or improving the normal tissue sparing (RefDA10 and RefDA12).

To evaluate the different plans, the following DVH derived parameters were obtained:

- coverage: $\mathrm{D}_{95}$, and $\mathrm{D}_{98}$;

- homogeneity: $\mathrm{D}_{20}, \mathrm{D}_{10}, \mathrm{D}_{2}$;

- effective dose: $\mathrm{D}_{\text {mean }}$ and $\mathrm{D}_{50}$;

- normal tissue: $\mathrm{V}_{12}$ and $\mathrm{V}_{10}$;

- conformity index: $C I=1+V_{\text {NTPD }} / V_{P T V P D}$, where $\mathrm{V}_{\text {NTPD }}$ is the volume (in $\mathrm{cm}^{3}$ ) of NT that receives a dose equal to or higher than the $\mathrm{PD}$, and $\mathrm{V}_{\mathrm{PTVPD}}$ the target volume (in $\mathrm{cm}^{3}$ ) that receives a dose equal to or higher that the PD.

For the specific case of VMAT, the $\mathrm{D}_{90}$ and $\mathrm{D}_{\text {mean }}$ of each inner auxiliary volume have been scored.

The original treatment plans used for patients had a slightly different $\%$ of target volume than the PD. To make a meaningful comparison, all DA plans were normalized to $\mathrm{D}_{95}$ equal to the prescription dose, $\mathrm{PD}$, and then the VMAT plans were optimized taking into account this reference.

\section{Results and discussion}

In Table 2 the results are presented for DA and VMAT for the 5 cases evaluated. The VMAT results were significantly superior in NT sparing $\mathrm{V}_{10}$ and $\mathrm{V}_{12}$ in all cases, with a $2-3 \mathrm{~cm}^{3}$ saving in each. In addition, VMAT improves the dose coverage $\mathrm{D}_{95}$ by about $0.5 \mathrm{~Gy}$. The objective of dose escalation is obtained by VMAT with a huge increment, about 2 Gy of the tumor mean and median dose. As a consequence of the NT sparing improvement, the CI was also significantly better for the VMAT plans in all cases. The encouraging general result is that when comparing VMAT vs DA, the VMAT improves the
NT sparing, and target coverage while allowing dose escalation.

To illustrate the comparison, isodoses on a slice are shown in Figure 1 for both plans for the highest, lowest and medium volume metastasis. In addition, the auxiliary inner volumes are shown. It can be seen how VMAT allows for higher conformity and dose scaling following the auxiliary volumes. In Figure 2, a DVH comparison is presented for one case only for illustrative purposes. The NT and target for DA and VMAT are presented. VMAT provides superior NT sparing plus conformity and dose scaling. Dose escalation follows the inner auxiliary volumes as can be seen in their DVHs. In Table 3, the resulting auxiliary inner structures volume are detailed, together with the representative dose for each one $\left(D_{90}\right.$ and $\left.D_{\text {mean }}\right)$, showing that dose intensification follows the modulation according to subsequent inner volumes.

Finally, for case \#5 with the lower prescription dose with DA due to its size $\left(6.66 \mathrm{~cm}^{3}\right)$, another VMAT plan was created in which the dose was increased to the NT $V_{10}$ and $V_{12}$ parameters obtained in DA, RefDA10 and RefDA12. The final $V_{10}$ and $\mathrm{V}_{12}$ were $12.84 \mathrm{~cm}^{3}$ and $8.56 \mathrm{~cm}^{3}$, respectively, versus the DA corresponding values of $13.48 \mathrm{~cm}^{3}$ and $9.08 \mathrm{~cm}^{3}$. In this "enlarged" plan the prescription dose was increased from the $\mathrm{D}_{95}$ of $16 \mathrm{~Gy}$ for DA plan to $\mathrm{D}_{97}$ of $18 \mathrm{~Gy}$ for VMAT, together with a very high mean and median dose of 21.6 Gy and 21.7 Gy, respectively. DVHs for both plans are compared in Figure 3. This illustrates the potential to scale the dose using VMAT that could be of major interest in cases of relatively radioresistant metastases such as melanoma, sarcoma or renal ones.

A dose-volume response relationship has been demonstrated for BM treated with SRS. In addition, mean dose per volume is strongly predictive of local control [27]. The possibility of dose escalation in in-

Table 2. Dosimetric parameters comparison of dynamic conformal arcs (DA)/(VMAT) plans for the 5 cases. $D_{x}$ parameters for the tumor (in Gy) and Vx for normal tissue (in $\mathrm{cm}^{3}$ ). Conformity index is also included

\begin{tabular}{|l|c|c|c|c|c|c|c|c|c|c|c|}
\hline Case\# & $D_{95}$ & \multicolumn{1}{c}{$\mathrm{D}_{98}$} & \multicolumn{1}{c}{$\mathrm{D}_{20}$} & \multicolumn{1}{c}{$\mathrm{D}_{10}$} & $\mathrm{D}_{2}$ & $\mathrm{D}_{\text {mean }}$ & $\mathrm{D}_{50}$ & NTV $_{10}$ & NTV $_{12}$ & $\mathrm{CI}$ \\
\hline 1 & $18 / 18.5$ & $17.6 / 17.6$ & $20.2 / 23.0$ & $20.3 / 23.6$ & $20.5 / 24.0$ & $19.5 / 21.4$ & $19.7 / 21.5$ & $11.38 / 8.02$ & $7.77 / 5.04$ & $1.278 / 1.097$ \\
\hline 2 & $18 / 18.5$ & $17.7 / 17.7$ & $20.3 / 24.4$ & $20.6 / 24.9$ & $20.8 / 25.5$ & $19.6 / 22.2$ & $19.7 / 22.5$ & $7.02 / 4.81$ & $4.85 / 3.05$ & $1.162 / 1.049$ \\
\hline 3 & $18 / 18.3$ & $17.6 / 17.6$ & $20.4 / 23.8$ & $20.6 / 25.1$ & $20.8 / 25.8$ & $19.6 / 21.5$ & $19.8 / 21.2$ & $11.61 / 8.28$ & $8.08 / 5.25$ & $1.267 / 1.063$ \\
\hline 4 & $17.5 / 18.1$ & $17.1 / 17.3$ & $19.8 / 23.4$ & $20.1 / 24.5$ & $20.3 / 25.3$ & $19.1 / 21.2$ & $19.2 / 21.0$ & $8.64 / 5.95$ & $6.07 / 3.82$ & $1.249 / 1.087$ \\
\hline 5 & $16 / 16.6$ & $15.5 / 15.7$ & $18.3 / 21.4$ & $18.4 / 22.4$ & $18.6 / 23.6$ & $17.6 / 19.5$ & $17.8 / 19.2$ & $8.88 / 5.85$ & $5.98 / 3.69$ & $1.278 / 1.101$ \\
\hline
\end{tabular}




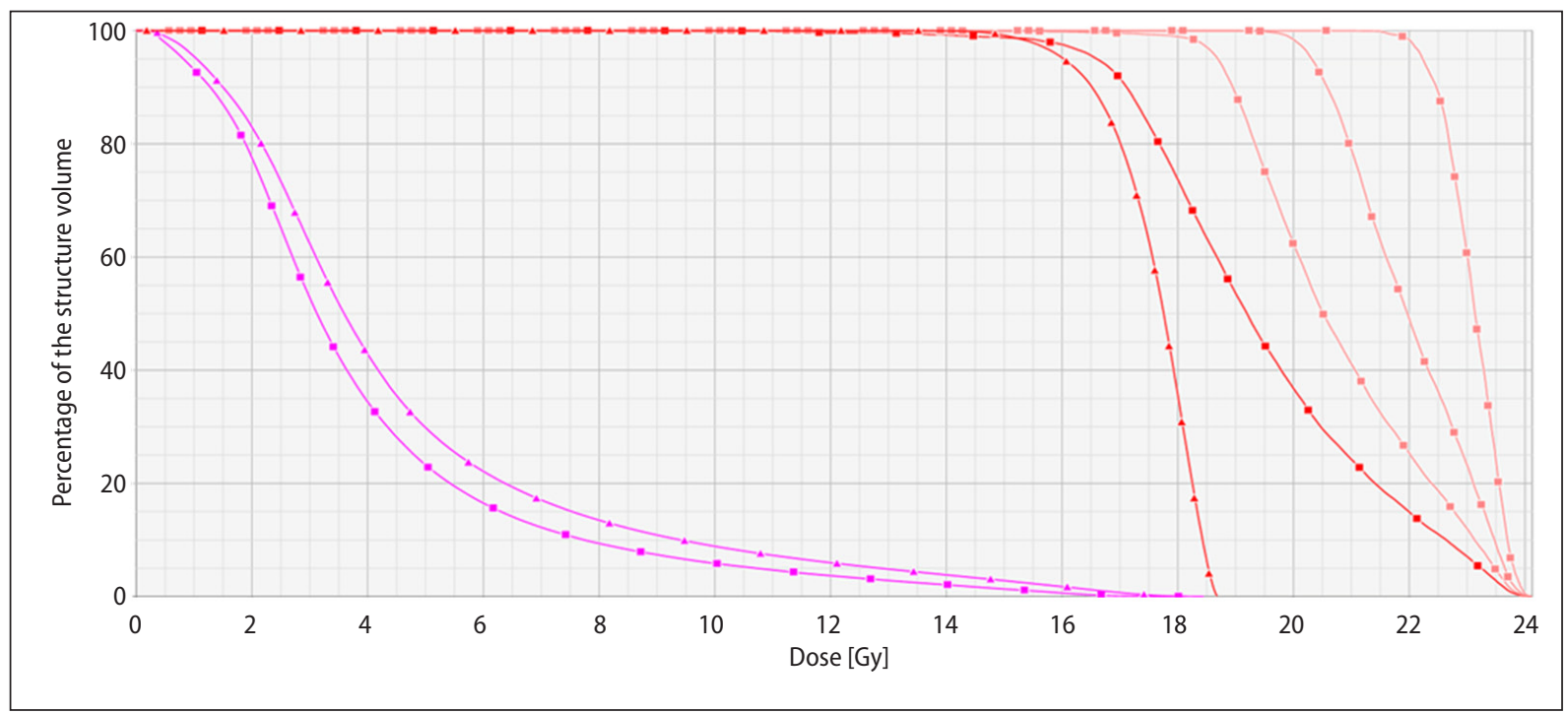

Figure 2. DVH comparison of DA plan (triangle) vs. VMAT (square) for case \#6. Normal tissue (magenta), target (red) and inner auxiliary volumes (pink) are shown. PD was 16 Gy for this case

Table 3. Volume $\left(\mathrm{cm}^{3}\right)$ of lesions and auxiliary inner structures together with $D_{90}$ and $D_{\text {mean }}(G y)$ in each auxiliary structure

\begin{tabular}{|l|c|c|c|c|c|c|c|}
\hline Case \# & Vol & \multicolumn{1}{c}{ Vol C1 } & Vol C2 & \multicolumn{1}{c|}{ Vol C3 } & $D_{90} / D_{\text {mean }}$ C1 & $D_{90} / D_{\text {mean }}$ C2 & $D_{90} / D_{\text {mean }}$ C3 \\
\hline 1 & 4.31 & 2.19 & 0.71 & NA & $21.5 / 22.7$ & $22.7 / 23.5$ & NA \\
\hline 2 & 4.48 & 2.73 & 1.50 & 0.67 & $22.0 / 23.7$ & $23.5 / 24.5$ & $24.4 / 25.0$ \\
\hline 3 & 5.55 & 3.38 & 1.90 & 0.91 & $20.9 / 22.9$ & $22.6 / 24.1$ & $24.4 / 25.2$ \\
\hline 4 & 5.70 & 3.76 & 1.89 & 0.89 & $20.4 / 22.4$ & $22.2 / 23.7$ & $24.0 / 24.7$ \\
\hline 5 & 6.66 & 3.93 & 2.02 & 0.78 & $18.9 / 20.8$ & $20.6 / 22.0$ & $22.5 / 23.1$ \\
\hline
\end{tabular}

dividual cases can be very useful during the evaluation of the most radical SRS dosimetry, while at the same time the dose constraints are kept within the established criteria.

During recent years, there has been a trend toward frameless SRS treatments. Nevertheless, care must be taken with NT irradiation in those patients with BM within eloquent areas, or close to the optic pathway or brainstem, due to the extra set-up margin. In our current practice, if frameless treatment is used, the VMAT technique is always employed because the treatment planning and verification can be done in advance. VMAT is also used in SRS with a frame for benign cases as Schwannoma due to the irregular shape and OAR vicinity.

Until now, the established approach in our departments for frame-based SRS in small size metastases has been the DA technique. The present study was motivated to evaluate the benefit of conformity even in these small lesions. This potential VMAT advantage together with the dose escalation op- tion is promoting the current technique reevaluation in our departments. The use of VMAT in SRS frame-based cases has an impact on workflow and resources, VMAT planning takes more time than the DA and the VMAT verification must be included in the workflow. This verification requires specific preparation, phantom set-up (in our case the Octavius 4D with the high definition array Octavius Detector 1000 SRS [PTW, Freiburg, Germany]) and analysis.

In frame-based SRS, there are situations in which a reduction of NT is required. Usually, in the DA technique we have used a $1 \mathrm{~mm}$ MLC margin; the reduction of this margin (by $0.5 \mathrm{~mm}$ for instance) improves NT sparing but, consequently, the dose inhomogeneity increases. For some cases, we have seen that it is also possible to do a correction of dose distribution inside the tumor by using field-in-field compensation along with some arc frames. However, this DA technique cannot manage concave shapes of the target as VMAT can. 


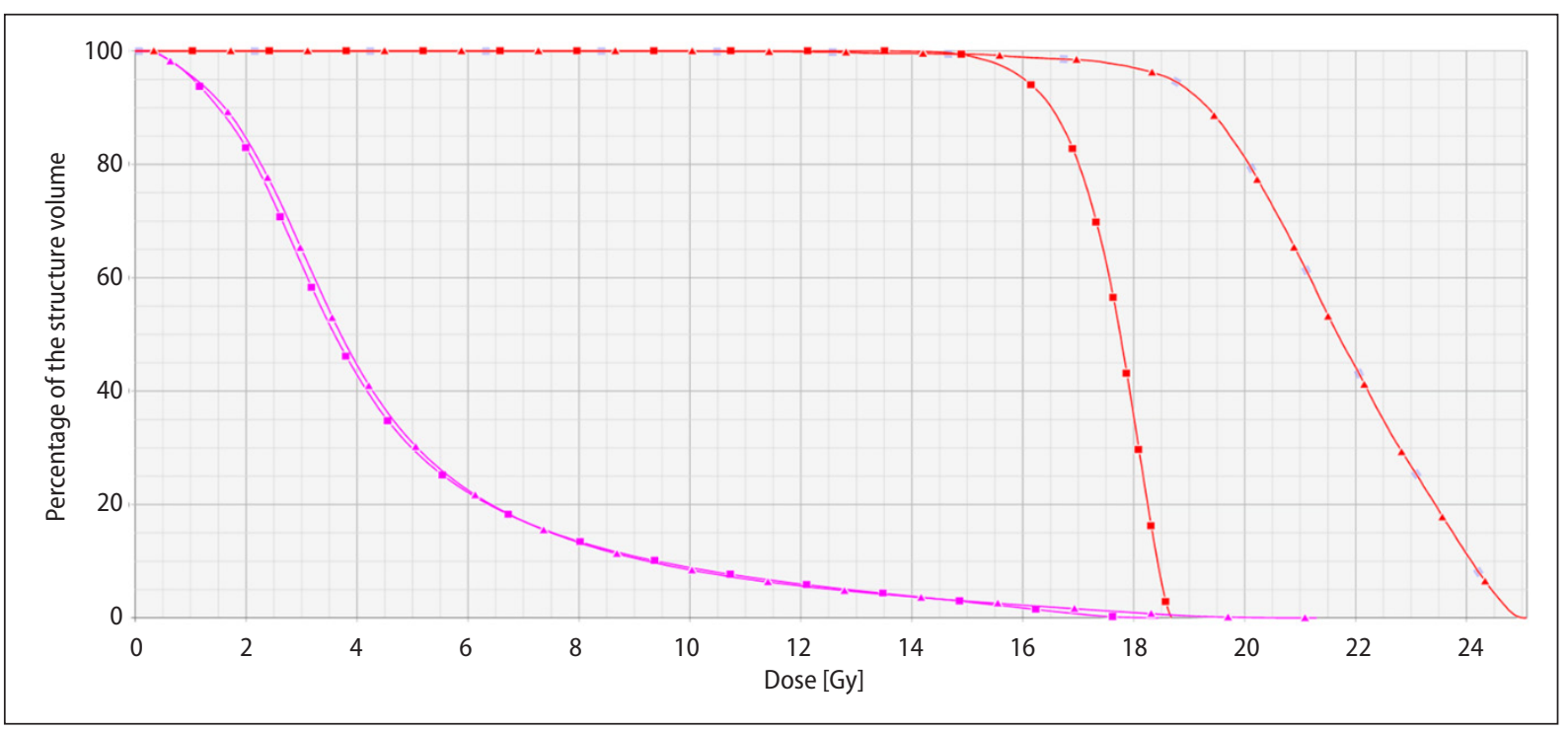

Figure 3. DVH comparison of DA plan (red squares) for case \#5 with VMAT plan (red triangle) with nearly the same values for normal tissue (magenta squares and triangles) $V_{10}$ and $V_{12}$. Dose to the tumor (red) can be increased from the prescribed dose in DA plan of $D_{95} 16 \mathrm{~Gy}$ to $18 \mathrm{~Gy}$ for $\mathrm{D}_{97}$ for the VMAT case

According to the target shape, it is reasonable to decide between DA and VMAT based on the commented benefits.

This study has been limited to just 5 cases, ranging through different volumes, treated with DA. This limited number was selected because of the illustrative purpose of the work regarding the role of VMAT. The NT values of all these DA cases are representative ones; typically, the plans resulted with $V_{10}$ and $V_{12}$ lower than our limit values of 15 $\mathrm{cm}^{3}$ and $10 \mathrm{~cm}^{3}$, respectively, reducing the risk of radionecrosis.

The selection of an increment of $2 \mathrm{~Gy} / \mathrm{mm}$ through the inner auxiliary volumes has been arbitrary with the sole aim of exploring the scaling capabilities. No other values were tested. It should be added that the final tuning NT sparing vs target coverage (and then the specific $\mathrm{V}_{10}$ and $\mathrm{V}_{12}$ resulting values) in VMAT included some subjectivity derived from the competivity in the objective function.

The VMAT procedure has been fully described with the aim of being planner-independent. Auxiliary shell design procedure and dose gradient have been well described; moreover, the optimization parameters have been included in detail in Table 1. However, it is necessary to add that these are specific for the TPS used (Eclipse) and even the version (v. 15.5) and probably should be adapted according to other TPS and versions.
The VMAT technique for SRS BM is not new [19]. In our study, we tried to demonstrate the feasibility to manage this technique in both dose conformation, then NT sparing, and modulation through the lesion center with dose inhomogeneity control through auxiliary shells. The presented method based on VMAT has been compared using only the DA technique. Cones were also used classically to treat $\mathrm{BM}$, but with the small leaf width MLC availability, the higher conformity of DA and IMRT-VMAT have been imposed.

Although this study has been focused on SRS frame-based cases, in our opinion it is also applicable to frameless cases in both SRS and HFSRT in which the inner auxiliary volumes and steep gradients could be adapted. This method, and other tools for dose scaling, could enable a more effective and safe application of SRS-HFSRT to large volume or tumors with complex spatial configuration.

Munshi et al. [30] evaluated the dose fall-off pattern for linear accelerator based on frameless stereotactic radiotherapy and radiosurgery using two different techniques, three-dimensional conformal radiotherapy and volumetric modulated arc therapy. Their results showed similar 100-80\%, $100-50 \%, 100-20 \%$ distance values. Although they used conformal beams instead of DA, these results seem in some contradiction with ours. In our opinion, the NT sparing improvement comes from the higher conformity to the target surface available 
with VMAT, even for these small volume targets. This is what we have tried to demonstrate in this work.

\section{Conclusions}

In this work, feasibility and potential benefit of VMAT in SRS treatment of the $4-6 \mathrm{~cm}^{3}$ size metastasis with respect to NT sparing and internal dose escalation has been demonstrated. A practical VMAT optimization procedure, based on auxiliary inner volumes, for dose scaling has been developed in order to minimize planner-dependence.

\section{Conflict of interests}

Authors declare that there is no conflict of interests regarding the publication of this paper.

\section{Funding}

None declared.

\section{References}

1. Posner JB, Chernik NL. Intracranial metastases from systemic cancer. Adv Neurol. 1978; 19: 579-592, indexed in Pubmed: 570349.

2. Patchell R. The management of brain metastases. Cancer Treat Rev. 2003; 29(6): 533-540, doi: 10.1016/s03057372(03)00105-1, indexed in Pubmed: 14585263.

3. Nussbaum ES, Djalilian HR, Cho KH, et al. Brain metastases. Histology, multiplicity, surgery, and survival. Cancer. 1996; 78(8): 1781-1788, indexed in Pubmed: 8859192.

4. Bradley KA, Mehta MP. Management of brain metastases. Semin Oncol. 2004; 31(5): 693-701, doi: 10.1053/j.seminoncol.2004.07.012, indexed in Pubmed: 15497123.

5. Grunert M, Kassubek R, Danz B, et al. Radiation and Brain Tumors: An Overview. Crit Rev Oncog. 2018; 23(1-2): 119-138, doi: 10.1615/CritRevOncog.2018025927, indexed in Pubmed: 29953371.

6. Minniti G, Filippi AR, Osti MF, et al. Radiation therapy for older patients with brain tumors. Radiat Oncol. 2017; 12(1): 101, doi: 10.1186/s13014-017-0841-9, indexed in Pubmed: 28629376.

7. Thomas SS, Dunbar EM. Modern multidisciplinary management of brain metastases. Curr Oncol Rep. 2010; 12(1): 34-40, doi: 10.1007/s11912-009-0073-8, indexed in Pubmed: 20425606.

8. Videtic GMM, Gaspar LE, Aref AM, et al. Expert Panel on Radiation Oncology-Brain Metastases. American College of Radiology appropriateness criteria on multiple brain metastases. Int J Radiat Oncol Biol Phys. 2009; 75(4): 961-965, doi: 10.1016/j.ijrobp.2009.07.1720, indexed in Pubmed: 19857783.

9. Linskey ME, Andrews DW, Asher AL, et al. The role of stereotactic radiosurgery in the management of patients with newly diagnosed brain metastases: a systematic review and evidence-based clinical practice guideline. J
Neurooncol. 2010; 96(1): 45-68, doi: 10.1007/s11060-0090073-4, indexed in Pubmed: 19960227.

10. Kocher M, Wittig A, Piroth MD, et al. Stereotactic radiosurgery for treatment of brain metastases. A report of the DEGRO Working Group on Stereotactic Radiotherapy. Strahlenther Onkol. 2014; 190(6): 521-532, doi: 10.1007/ s00066-014-0648-7, indexed in Pubmed: 24715242.

11. Mehta MP, Tsao MN, Whelan TJ, et al. The American Society for Therapeutic Radiology and Oncology (ASTRO) evidence-based review of the role of radiosurgery for brain metastases. Int J Radiat Oncol Biol Phys. 2005; 63(1): 37-46, doi: 10.1016/j.ijrobp.2005.05.023, indexed in Pubmed: 16111570.

12. Sallabanda M, García-Berrocal MI, Romero J, et al. Hypofractionated Re-irradiation after Maximal Surgical Resection for Recurrent Glioblastoma: Therapeutic Adequacy and Its Prognosticators of Survival. Yonsei Med J. 2018; 59(2): 194-201, doi: 10.3349/ymj.2018.59.2.194, indexed in Pubmed: 29436186.

13. Tsao M, Xu W, Sahgal A. A meta-analysis evaluating stereotactic radiosurgery, whole-brain radiotherapy, or both for patients presenting with a limited number of brain metastases. Cancer. 2012; 118(9): 2486-2493, doi: 10.1002/ cncr.26515, indexed in Pubmed: 21887683.

14. Chang EL, Wefel JS, Hess KR, et al. Neurocognition in patients with brain metastases treated with radiosurgery or radiosurgery plus whole-brain irradiation: a randomised controlled trial. Lancet Oncol. 2009; 10(11): 1037-1044, doi: 10.1016/S1470-2045(09)70263-3, indexed in Pubmed: 19801201.

15. Higuchi $Y$, Yamamoto M, Serizawa T, et al. Modern management for brain metastasis patients using stereotactic radiosurgery: literature review and the authors' gamma knife treatment experiences. Cancer Manag Res. 2018; 10: 1889-1899, doi: 10.2147/CMAR.S116718, indexed in Pubmed: 30013395.

16. Barbour $A B$, Jacobs $C D$, Williamson $H$, et al. Radiation Therapy Practice Patterns for Brain Metastases in the United States in the Stereotactic Radiosurgery Era. Adv Radiat Oncol. 2020; 5(1): 43-52, doi: 10.1016/j.adro.2019.07.012, indexed in Pubmed: 32051889.

17. Nieder C, Grosu AL, Gaspar LE. Stereotactic radiosurgery (SRS) for brain metastases: a systematic review. Radiat Oncol. 2014; 9: 155, doi: 10.1186/1748-717X-9-155, indexed in Pubmed: 25016309.

18. Hazard LJ, Wang B, Skidmore TB, et al. Conformity of LINAC-based stereotactic radiosurgery using dynamic conformal arcs and micro-multileaf collimator. Int J Radiat Oncol Biol Phys. 2009; 73(2): 562-570, doi: 10.1016/j.ijrobp.2008.04.026, indexed in Pubmed: 18565688.

19. Teoh M, Clark CH, Wood K, et al. Volumetric modulated arc therapy: a review of current literature and clinical use in practice. Br J Radiol. 2011; 84(1007): 967-996, doi: 10.1259/ bjr/22373346, indexed in Pubmed: 22011829.

20. Roa DE, Schiffner DC, Zhang J, et al. The use of RapidArc volumetric-modulated arc therapy to deliver stereotactic radiosurgery and stereotactic body radiotherapy to intracranial and extracranial targets. Med Dosim. 2012; 37(3): 257-264, doi: 10.1016/j.meddos.2011.09.005, indexed in Pubmed: 22365418.

21. Grimm MA, Köppen U, Stieler F, et al. Prospective assessment of mask versus frame fixation during Gamma Knife 
treatment for brain metastases. Radiother Oncol. 2020; 147: 195-199, doi: 10.1016/j.radonc.2020.05.011, indexed in Pubmed: 32416280.

22. Minniti G, ClarkeE, Lanzetta G, et al.Stereotactic radiosurgery for brain metastases: analysis of outcome and risk of brain radionecrosis. Radiat Oncol. 2011; 6: 48, doi: 10.1186/1748717X-6-48, indexed in Pubmed: 21575163.

23. Sneed PK, Mendez J, Vemer-van den Hoek JGM, et al. Adverse radiation effect after stereotactic radiosurgery for brain metastases: incidence, time course, and risk factors. J Neurosurg. 2015; 123(2):373-386, doi: 10.3171/2014.10. JNS141610, indexed in Pubmed: 25978710.

24. Korytko T, Radivoyevitch T, Colussi V, et al. 12 Gy gamma knife radiosurgical volume is a predictor for radiation necrosis in non-AVM intracranial tumors. Int J Radiat Oncol Biol Phys. 2006; 64(2): 419-424, doi: 10.1016/j. ijrobp.2005.07.980, indexed in Pubmed: 16226848.

25. Blonigen BJ, Steinmetz RD, Levin L, et al. Irradiated volume as a predictor of brain radionecrosis after linear accelerator stereotactic radiosurgery. Int J Radiat Oncol Biol Phys. 2010; 77(4): 996-1001, doi: 10.1016/j.jjrobp.2009.06.006, indexed in Pubmed: 19783374.

26. Hong LX, Garg M, Lasala P, et al. Experience of micromultileaf collimator linear accelerator based single fraction stereotactic radiosurgery: tumor dose inhomo- geneity, conformity, and dose fall off. Med Phys. 2011; 38(3): 1239-1247, doi: 10.1118/1.3549764, indexed in Pubmed: 21520836.

27. Amsbaugh MJ, YusufMB, Gaskins J, et al. A Dose-Volume Response Model for Brain Metastases Treated With Frameless Single-Fraction Robotic Radiosurgery: Seeking to Better Predict Response to Treatment. Technol Cancer Res Treat. 2017; 16(3): 344-351, doi: 10.1177/1533034616685025, indexed in Pubmed: 28027696.

28. Marcrom SR, McDonald AM, Thompson JW, et al. Fractionated stereotactic radiation therapy for intact brain metastases. Adv Radiat Oncol. 2017; 2(4): 564-571, doi: 10.1016/j. adro.2017.07.006, indexed in Pubmed: 29204523.

29. Grimm MA, Köppen U, Stieler F, et al. Prospective assessment of mask versus frame fixation during Gamma Knife treatment for brain metastases. Radiother Oncol. 2020; 147: 195-199, doi: 10.1016/j.radonc.2020.05.011, indexed in Pubmed: 32416280.

30. Munshi A, Sarkar B, Roy S, et al. Dose fall-off patterns with volumetric modulated arc therapy and three-dimensional conformal radiotherapy including the "organ at risk" effect. Experience of linear accelerator-based frameless radiosurgery from a single institution. Cancer Radiother. 2019; 23(2): 138-146, doi: 10.1016/j.canrad.2018.10.003, indexed in Pubmed: 30797691. 